\title{
Documenting Digital Creative Practice
}

\author{
Rehan Zia \\ Bournemouth University \\ Poole, United Kingdom \\ rzia@bournemouth.ac.uk
}

\begin{abstract}
This paper is grounded in the author's experience of documenting his own digital high dynamic range landscape photography and visual effects practices, and, the advantages, value, limitations and impact this has on the creative practice and vice versa. Documenting of digital creative practices is becoming increasingly important for creative businesses, practitioners, researchers and academics. For photographers, filmmakers, animators and visual effects artists, it is means of demonstrating innovative approaches to engage the public and prospective clients. For academics and researchers involved in practice-led research, documenting practice provides material for reflection and case studies as well as a means of evidencing the research journey and key decisions taken along the way. This paper will explore and compare different means, methods and approaches to documenting digital camera acquisition, processing and postproduction workflows depending on the audience consumption and usage - i.e. self, private or public, and utility i.e. selfreflective practice, communication of insights, evidencing and cataloguing research and creating case studies. A range of approaches from hand written notes, photography to a number of different video documentation approaches will be discussed and evaluated in terms of ease of documentation, situational constraints, impact on the creative practice, ease of storage, and, dissemination.
\end{abstract}

Documenting practice. Video documentation. Practice-led.

\section{INTRODUCTION}

There is an increasing need for researchers involved in practice research to document and provide commentary on their practice (Candy \& Edmonds 2011) in order to evidence, analyse, reflect upon and/or communicate research insights. Professional practitioners, similarly, may want to demonstrate novel workflows and techniques to inspire others, share practice insights, impress prospective clients and/or market ones' services and skills. Depending on the intent, goal, motivation and target audience, the nature, approach and method of documentation may vary.

This paper explores various different documentation approaches - primarily video-based - that have been utilised by the author over the course of their $\mathrm{PhD}$ and on-going professional practice. The main focus of the video recording, documentation and commentary was the craft production process. As such, it was important to record both changes in the scene being photographed, as well as the photographers' response and adaptation to the changing scene conditions, i.e. the photographer's performance.

\section{INTENT}

Over the course of the PhD research, the intention behind documenting the landscape photography practice was to use it - alongside the photographs captured - as a means of critical reflection and analysis to explore best practice for stack-based high dynamic range landscape photography (Zia 2020). The documentation would also serve as evidence of the research process and demonstrate the iterative improvements in craft technique and workflow best practices brought about through critical thinking.

Video documentation was also subsequently used as a means of providing insights into professional practice, and, encouraging students and amateur photographers to experiment with the techniques and workflow.

\section{DOCUMENTING THE "KNOW-WHAT"}

The digital photograph metadata inherently records the camera and lens make and model along with exposure settings such as the exposure time, aperture value and ISO. This information - though useful in itself - can also be used to deconstruct the 
light and object surface luminance values in the scene. Thus, data about the parameters used to capture the digital photograph is recorded easily enough. However, the critical reflection that informs decisions leading up to the moment of the photographic capture, or what Nelson (2013, p. 44) describes as the "know-what", can be far more important and need to be captured explicitly. These decisions may include key considerations about framing, composition, motion and lighting changes within the scene, as well as physical and logistical constraints of the environment, i.e. why the photographer made the decisions they did instead of choosing other alternatives (Lumet 1995, p. ix). Whist the camera metadata records what parameter choices were made, it does not record the decision-making process that led to those choices.

This problem is not limited to digital photographs only. 3D models can retain the series of operations, or, "construction history" that led to the final form but not the maker's critical decision making over the choice of one method or technique over another. Similarly, non-destructive digital image editing workflows and image editing history allow for step-by-step documentation of the image creation process to be recorded but the critical decision making process of the image editor needs to be recorded explicitly if needed.

\section{DOCUMENTATION APPROACHES}

\subsection{Image metadata}

The early documentation process relied entirely on the camera metadata. Whilst this was useful as a means for self-reflective practice, over time it proved insufficient as it failed to document scene conditions or alternative choices that would be useful to convey to other practitioners. It was also difficult to remember exactly what the scene conditions were over time. For some manual lenses, it is also not possible to automatically record the lens aperture and focus parameter data to the image metadata.

\subsection{Handwritten notes}

Making handwritten notes proved to be very tedious and intrusive as it took a long time to write and the depth in the scene descriptions was still found to be insufficient to convey all the decision parameters.

Writing in a notebook during wet and/or windy weather situations was particularly challenging and the attempt to write notes during photography excursions was quickly abandoned as they proved to be a great distraction from the actual practice of photography.

\subsection{Video documentation}

Video documentation approaches with the photographer thinking out loud and providing commentary on the decisions being made proved more successful. However, capturing this critical reflection and the performance/improvisation of the photographer to the changing elements in the scene alongside the scene itself proved more challenging.

\subsubsection{Using the main photography camera pre/post photography capture}

Using the photography camera to record quick videos before or after taking photographs proved useful in that the scene elements could be recorded along with voice notes/practitioner commentary on why particular decisions were being made (see Figure 1). The limitations with this method, however, were that, a) key photographic moments could be missed whilst recording the video commentary, b) the arrangement of the scene elements, the motion in the scene, and, the scene lighting could change between the time the photographs were captured and when the video was being recorded, and, c) it was not possible to record the photographer's improvisation and adaption to the changing scene conditions as it happened.

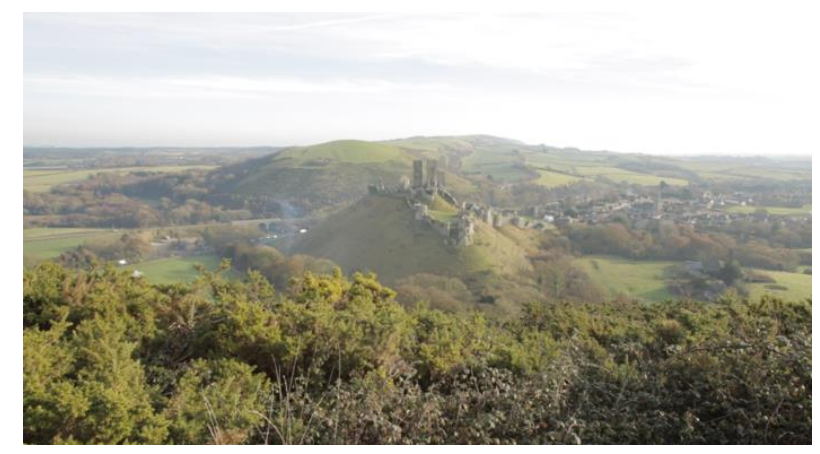

Figure 1: Recording video commentary postphotography using the same camera used for primary photography

\subsubsection{Secondary DSLR camera}

A secondary digital single-lens reflex camera on a separate tripod proved useful in capturing the photographer's performance and what was unfolding in the scene, albeit not always at the same time or in the same frame (see Figure 2). Wider angle lenses that captured the photography setup as well as the scene caused too much distortion and image elements further away would appear too small. Alternatively, a $50 \mathrm{~mm}$ focal length lens would not have a wide enough field of 
view to show both the practitioner and the scene in front of the camera.

The effort of carrying the additional camera and tripod over long walks and the extra setup/take down time made this approach unfeasible.

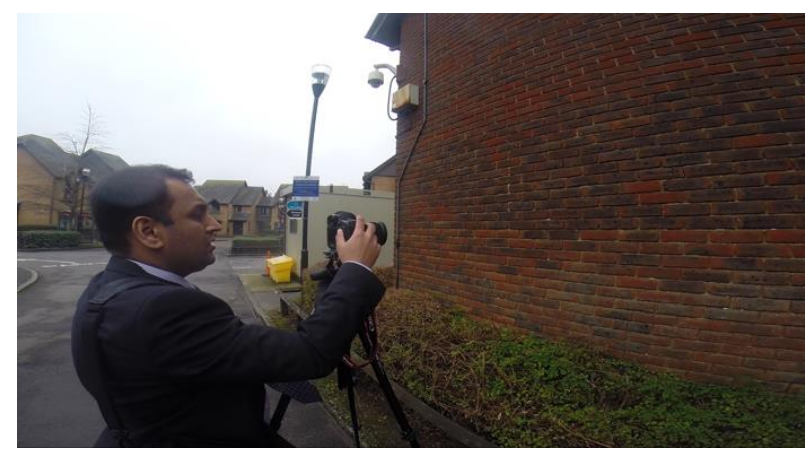

Figure 2: Video recording using a secondary camera on a separate tripod

\subsubsection{HD action camera in hotshoe}

A small HD action camera was used to document the scene being photographed along with the voice commentary by the photographer as decisions about the camera workflow, lens and exposure parameters were being made (see Figure 3). Whilst this approach was relatively non-intrusive in terms of the documentation workflow, it did add more wind resistance to the main camera resulting in greater camera shake and motion blur in the photographs and thus adversely affecting the practice results.

\subsubsection{HD action camera in headband}

In order to overcome the issue of wind resistance and camera shake, the HD action camera was used on a headband but the footage recorded was too shaky to the point of being rendered useless.

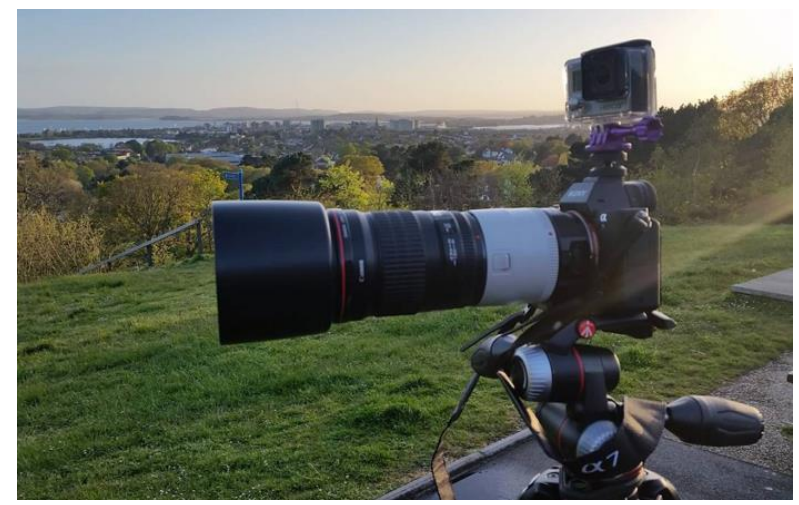

Figure 3: Video recording of the scene being photographed using a full HD action camera attached to main camera hotshoe with audio commentary by the photographer

\subsection{5. $360^{\circ}$ camera in hotshoe}

A $360^{\circ}$ camera attached to the main photography camera hotshoe proved quite successful in recording the entire environment being photographed as well as the actions of the photographer providing a more holistic view of the decision-making process and the photographer's performance (see Figure 4). Wind resistance and the risk of introducing motion blur in the photographs, however, limit the use of this approach to low wind scenarios.

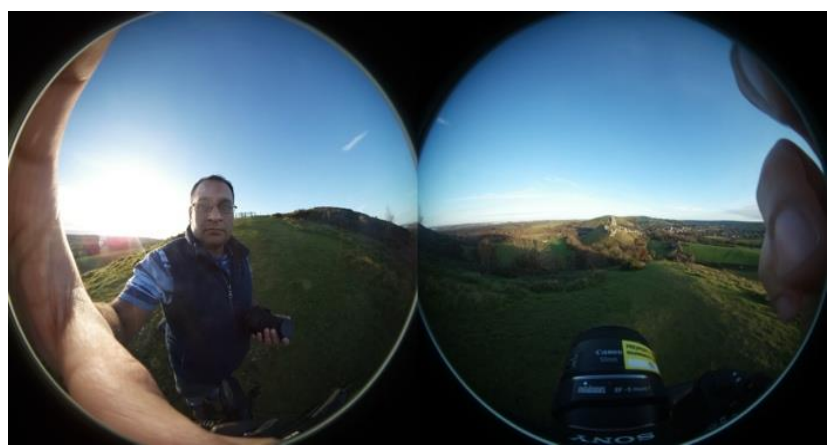

Figure 4: $360^{\circ}$ video recording allowing for video documenting the scene as well as the photographer's performance with the audio commentary

\subsubsection{Still images or video using camera phone}

Using the camera phone to take pictures, record video before or after photography - or sometimes during long exposures - proved to be a useful method for documenting and illustrating camera setups quickly (see Figures 5 - 8). The issue here was capturing the photographer's performance during the act of photography and taking attention away from the scene that may result in missing out on key photographic opportunities unfolding in the scene.

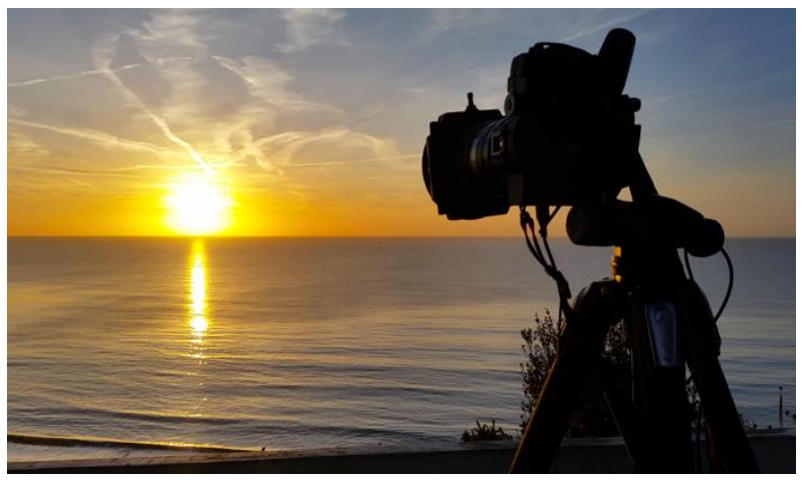

Figure 5: Video recording using camera phone during a long exposure illustrating the scene and camera setup 


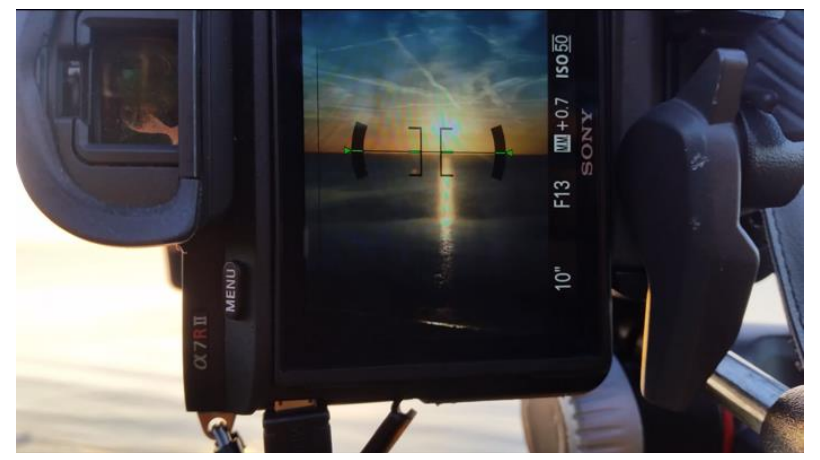

Figure 6: Video recording using camera phone during a long exposure illustrating the scene and camera setup

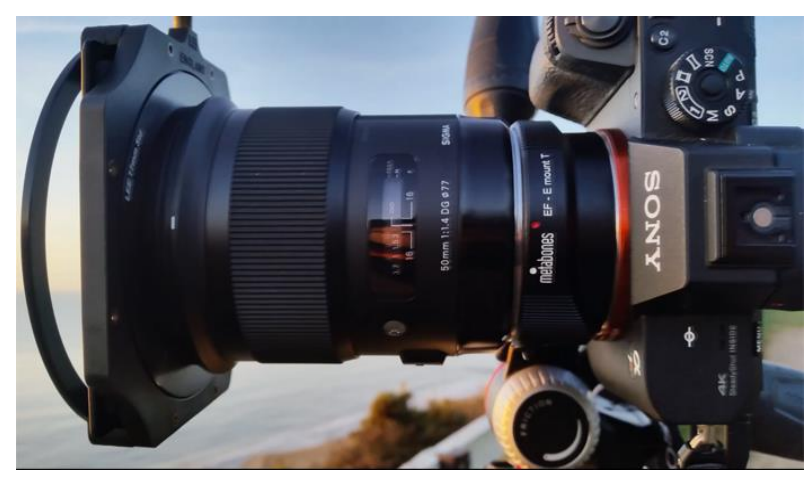

Figure 7: Video recording using camera phone during a long exposure illustrating the scene and camera setup

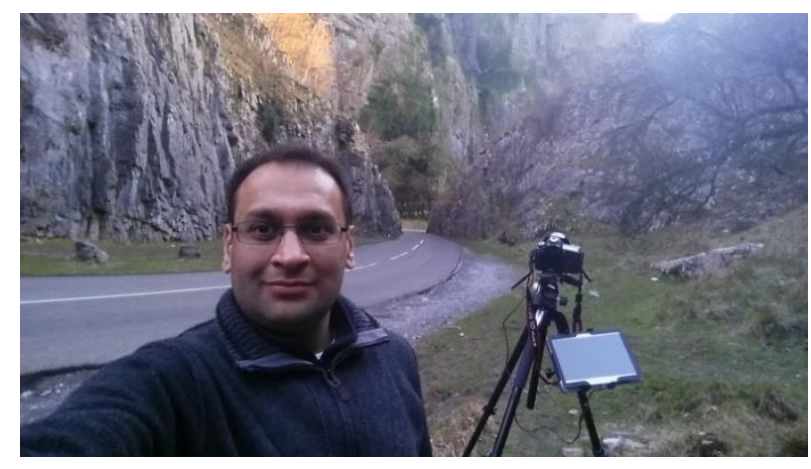

Figure 8: Photograph selfie showing camera setup and scene using camera phone during a long exposure illustrating the scene and camera setup

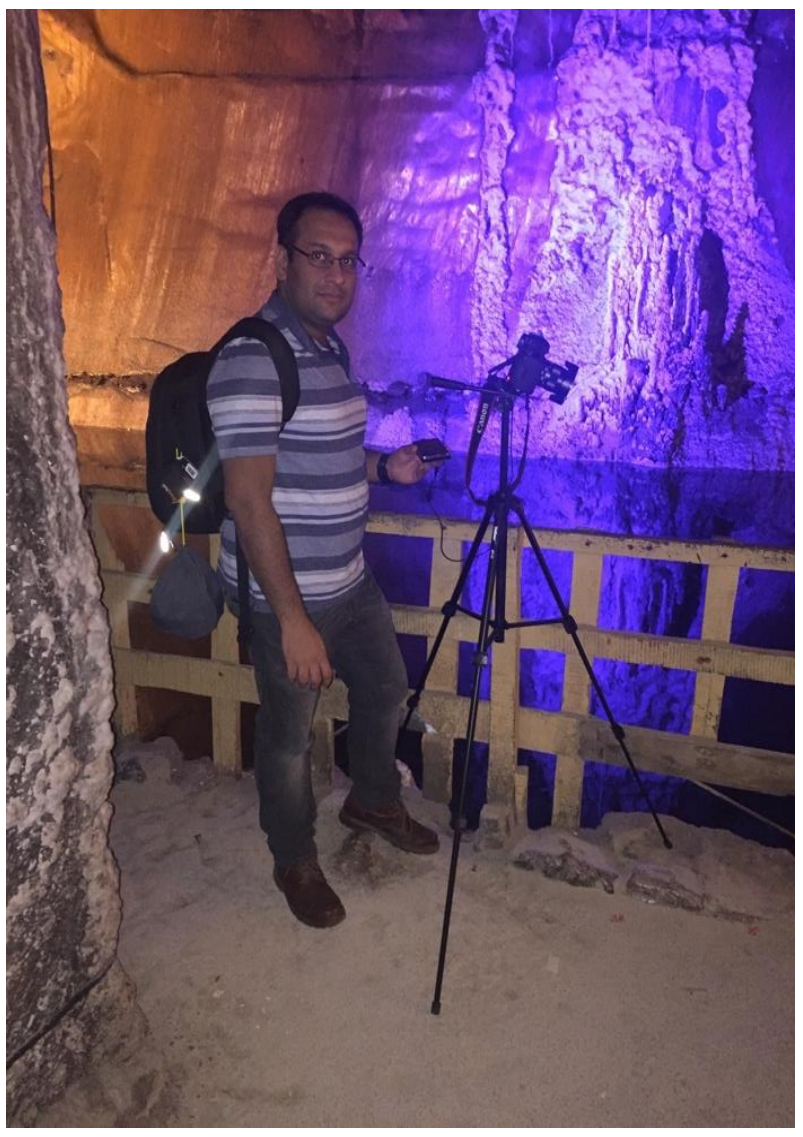

Figure 9: Photograph using camera phone (Courtesy of Daniyal Mirza)

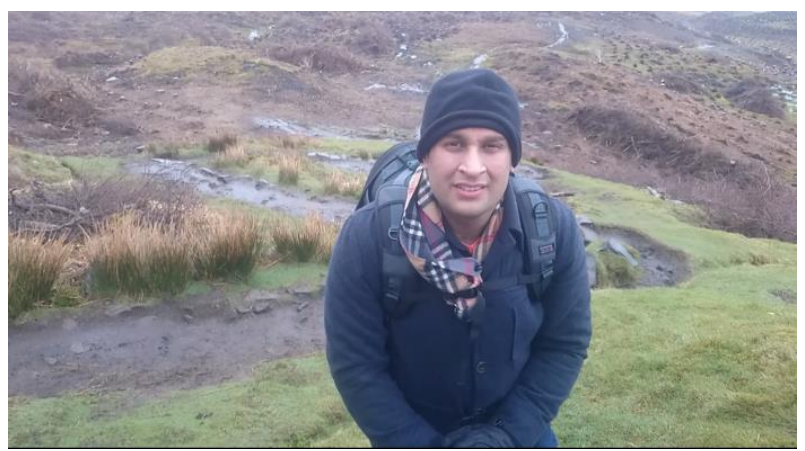

Figure 10: Video recording using camera phone (Courtesy of Fahd Sami) 


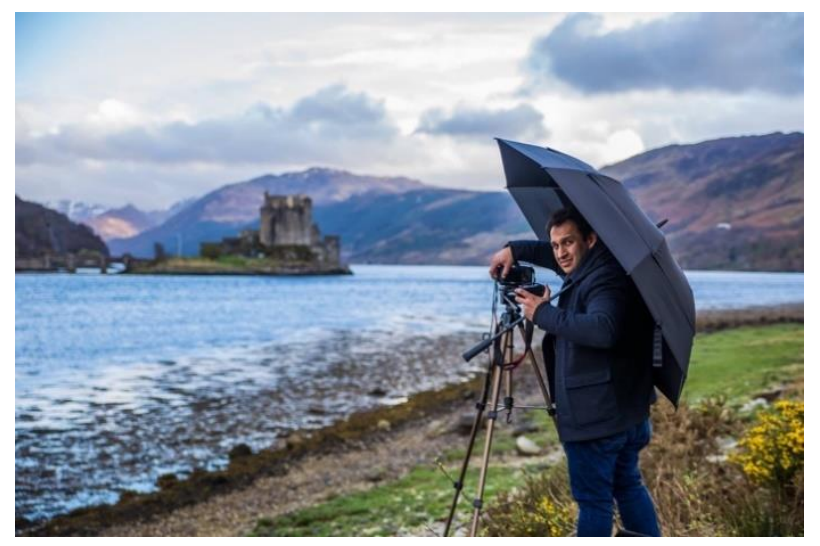

Figure 11: Photo recording using a DSLR camera (Courtesy of Fahd Sami)

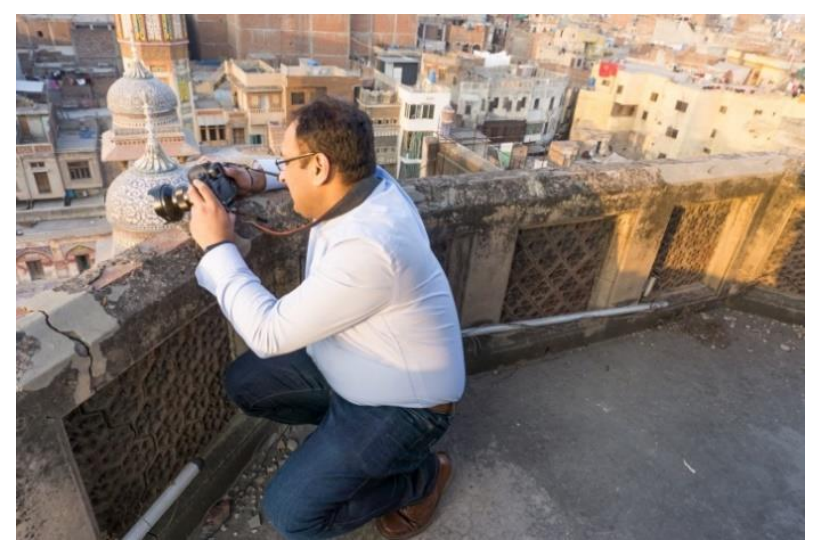

Figure 12: Photograph using secondary camera (Courtesy of Daniyal Mirza)

\subsubsection{Still images or video recorded by an assistant}

Having another person capture the photography performance and the scene using video and/or photographs has proved very useful in that the photographer is free to concentrate on the practice rather than worrying about the documentation process. The problem with this approach is that it can be expensive in case of a hired assistant, or, the quality of documentation may suffer if a nonexpert friend or family member is helping with the process.

\section{ISSUES AND ANALYSIS}

Nelson (2013, p. 87) notes that the process of documentation can not only result in excessive logistical demands but also interference with the practice itself.

The process of video and audio documentation can make the practitioner very self-conscious about being recorded which in turn can affect both the practice and the documentation.
Video documenting the practice requires not only extra recording equipment but also extra hard disk space and processing power in order to process the recordings.

Video documentation can also prove to be an intrusive process in that it may take focus away from the practice itself; this is especially problematic if the practitioner has to document and comment on their own practice and the work is time critical - as has been the case in the author's landscape photography practice. Energy, effort, resources and focus have to be divided between the actual practice, and, the documentation of that practice, that can adversely affect the practice, the documentation, or both.

\section{CONCLUSION AND FUTURE WORK}

Documenting the photographer's performance in relation to the changing scene, and, documenting the scene elements at the same time has proved to be a challenging exercise. However, the approaches discussed have had reasonable success to varying degree depending on the scene conditions and insights/commentary required for the target audience. Whilst expert practitioners may be able to infer the decisions being made by the photographer with relatively little information, it is important to have a more detailed commentary on the process if the target audience comprises of non-specialists.

Going forward, it would be interesting to see how a 360 camera could be used on a secondary tripod rather than attached to the photography camera hotshoe so as to avoid any wind resistance and camera shake.

\section{REFERENCES}

Candy, L. and Edmonds, E. (2011) The Role of the Artefact and Frameworks for Practice-Based Research. The Routledge Companion to Research in the Arts, (October 2010), pp. 1-18.

Lumet, S. (1995) Making movies. 1st edition. New York: Vintage Books.

Nelson, R. (2013) Practice as research in the arts: principles, protocols, pedagogies, resistances. Palgrave Macmillan.

Zia, R. (2020) Fixing it in the present-the decisive moment in high dynamic range landscape photography. Bournemouth University. 